\title{
On-line Ramsey Theory
}

\author{
J. A. Grytczuk and M. Hałuszczak \\ Faculty of Mathematics, Computer Science and Econometrics, University of Zielona Góra, \\ 65-516 Zielona Góra, Poland \\ J.Grytczuk@wmie.uz.zgora.pl, M.Haluszczak@wmie.uz.zgora.pl \\ H. A. Kierstead \\ Department of Mathematics and Statistics, Arizona State University, \\ Tempe, Arizona 85287, U.S.A. \\ Kierstead@asu.edu
}

Submitted: Apr 30, 2004; Accepted: Aug 19, 2004; Published: Sep 9, 2004

Mathematics Subject Classifications: 05C38, 15A15, 05A15, 15A18

\begin{abstract}
The Ramsey game we consider in this paper is played on an unbounded set of vertices by two players, called Builder and Painter. In one move Builder introduces a new edge and Painter paints it red or blue. The goal of Builder is to force Painter to create a monochromatic copy of a fixed target graph $H$, keeping the constructed graph in a prescribed class $\mathcal{G}$. The main problem is to recognize the winner for a given pair $H, \mathcal{G}$. In particular, we prove that Builder has a winning strategy for any $k$-colorable graph $H$ in the game played on $k$-colorable graphs. Another class of graphs with this strange self-unavoidability property is the class of forests. We show that the class of outerplanar graphs does not have this property. The question of whether planar graphs are self-unavoidable is left open. We also consider a multicolor version of Ramsey on-line game. To extend our main result for 3-colorable graphs we introduce another Ramsey type game, which seems interesting in its own right.
\end{abstract}

\section{Introduction}

In this paper we consider the following extension of the Ramsey on-line game introduced independently by Beck 1] and Friedgut et al. 22. Let $\mathcal{G}$ denote a class of finite graphs and let $H$ be a fixed graph from $\mathcal{G}$. There are two players-Builder and Painter - and the board of the game is an infinite, independent set of vertices. In each of her moves Builder presents a new edge and then Painter immediately colors it red or blue. Builder's goal is to force Painter to create a monochromatic copy of $H$, but there is a catch here; at 
any moment of the game (even after the very last move) the red-blue graph must belong to $\mathcal{G}$; otherwise the game stops and Builder loses. Thus, Builder wins the game if and only if she can force Painter to create a monochromatic copy of $H$ in a graph belonging to $\mathcal{G}$. In this case we say that $H$ is unavoidable (for Painter) in the Ramsey on-line game on $\mathcal{G}$. Otherwise we say that $H$ is avoidable.

Let $R(H)$ be a Ramsey graph of $H$, that is, a graph which exhibits a monochromatic copy of $H$ in any 2-coloring of its edges. If $R(H) \in \mathcal{G}$ then $H$ is trivially unavoidable on $\mathcal{G}$, but otherwise it may be difficult to detect the winner. Suppose, for instance, that $H=K_{4}$ and $\mathcal{G}$ consists of all 4-colorable graphs. Clearly, no Ramsey graph $R\left(K_{4}\right)$ belongs to $\mathcal{G}$, yet $K_{4}$ is unavoidable on this class. This is a special case of one of our results (Theorem 2 ) asserting that any $k$-colorable graph $H$ is unavoidable on $k$-colorable graphs. The fact that this result is based on two fundamental theorems of Ramsey theory, Ramsey's original unabridged theorem and the Bipartite Ramsey Theorem, may help convince the reader that we are indeed studying Ramsey theoretic questions.

It seems that this self-unavoidability of a class of graphs (not containing Ramsey graphs of its members) is a rather exceptional property. Therefore it would be interesting to find other non-trivial examples of this unexpected phenomenon. The last section offers a speculative discussion on this and related questions.

\section{$2 \quad k$-colorable graphs}

We start with some preparations for the proof of our first result. Let $[n]$ denote the set $\{1, \ldots, n\}$ and $\left(\begin{array}{l}X \\ k\end{array}\right)$ denote the set $\{S \subseteq X:|S|=k\}$. Let $R^{(k)}(u)$ be the smallest number $n$ (guaranteed to exist by Ramsey's theorem [3]) such that for every 2-coloring of $\left(\begin{array}{c}{[n]} \\ k\end{array}\right)$ there exists a $u$-subset $H \subseteq[n]$ such that $\left(\begin{array}{l}H \\ k\end{array}\right)$ is monochromatic.

Now we are ready to state and prove our first theorem. Note that in the following statement $n(k, k, k)$ is the value in which we are most interested.

Theorem 1 There exists a function $n(a, b, k)$ such that for all integers $a, b, k$ with $2 \leq$ $a, b \leq k$, Builder can force Painter to create either a red a-clique or a blue b-clique while building the edges of a $k$-colorable graph on $n(a, b, k)$ vertices.

Proof: We begin by recursively defining $n(a, b, k)$. Let

1. $n(2, i, k)=n(i, 2, k)=i$ for $2 \leq i \leq k$.

2. $n(a, b, k)=s(a, b, k)+t(a, b, k)$, where
(a) $s(a, b, k)=2(k-2)\left(\begin{array}{c}t(a, b, k) \\ k-1\end{array}\right) n(a-1, b-1, k-1)$,
(b) $t(a, b, k)=R^{(k-1)}(u(a, b, k))$ and
(c) $u(a, b, k)=\max \{n(a-1, b, k), n(a, b-1, k)\}$. 
We argue by induction on $a+b+k$. The base step is trivial so consider the induction step. We give Builder's strategy. Let $s=s(a, b, k), t=t(a, b, k)$ and $n=n(a, b, k)=s+t$. Let $V$ be a set of $n$ vertices and let $\{S, T\}$ be a partition of $V$ with $|S|=s$ and $|T|=t$, where $S$ has the form

$$
S=\left\{(F, i): F \in\left(\begin{array}{c}
T \\
k-1
\end{array}\right), i \in[2(k-2) n(a-1, b-1, k-1)]\right\} .
$$

First Builder presents all edges of the form $\{(F, i), j\}$, where $j \in F$. Notice that at this point Builder can still add any $(k-1)$-colorable graph $G$ to $S$ or any $k$-colorable graph $G^{\prime}$ to $T$ (but not both) and still have a $k$-colorable graph. The second statement follows from the fact that every vertex in $S$ has degree $k-1$ and so any $k$-coloring of $T$ can be extended to a $k$-coloring of $S \cup T$.

Case 1: For every $F \in\left(\begin{array}{c}T \\ k-1\end{array}\right)$ there exists $(F, i) \in S$ such that all edges incident to $(F, i)$ have the same color $c(F)$. This induces a coloring $c$ of the $(k-1)$-subsets of $T$ and, by the choice of $t$, there exists a $u$-subset $H \subseteq T$ such that $\left(\begin{array}{c}H \\ k-1\end{array}\right)$ is monochromatic under $c$ (say red). By the inductive hypothesis Builder can force painter to create either a red $(a-1)$-clique on some set $K$ or a blue $b$-clique, while building a $k$-colorable graph $G^{\prime}$ on $H$. In the latter case we are done. For the former case let $K \subseteq F \in\left(\begin{array}{c}H \\ k-1\end{array}\right)$. We can add $(F, i)$ to $K$ to get a red $a$-clique.

Case 2: There exists $F \in\left(\begin{array}{c}T \\ k-1\end{array}\right)$ such that every vertex $(F, i) \in S$ is incident to edges of both colors. Then there exists $x, y \in F$ and

$$
H \subseteq\{(F, i): i \in[2(k-2) n(a-1, b-1, k-1)]\}
$$

such that $|H|=n(a-1, b-1, k-1)$ and for all $z \in H$ we have $c(x z)=$ red and $c(y z)=$ blue. Indeed, fix a vertex $v \in F$. There exists $I \subseteq[2(k-2) n(a-1, b-1, k-1)]$ such that $|I| \geq(k-2) n(a-1, b-1, k-1)$ and the edges $v(F, i)$, with $i \in I$, are of the same color (say red). Set $x=v$. Also there exists $w \in F$ and $I^{\prime} \subseteq I$ such that $\left|I^{\prime}\right| \geq n(a-1, b-1, k-1)$ and the edge $w(F, i)$ is blue for every $i \in I^{\prime}$. Set $y=w$. Let $H=\left\{(F, i): i \in I^{\prime}\right\}$. By the induction hypothesis Builder can force painter to create either a red $(a-1)$-colorable clique or a blue $(b-1)$-clique, while building a $(k-1)$-colorable graph $G$ on $H$. In either case we are done by adding $x$ or $y$ to the clique.

We shall use the above result together with the Bipartite Ramsey Theorem (see [3]) to show that the class of $k$-colorable graphs is self-unavoidable. Let $B(p)$ be the smallest number $t$ (guaranteed to exist by the Bipartite Ramsey Theorem [3]) such that for every 2-edge-coloring of $K_{t, t}$ there exists a monochromatic copy of $K_{p, p}$. Let $B^{(m)}(p)$ be the $m$-fold iteration of $B(p)$.

A "blown-up" copy of a simple graph $G$ is a graph obtained by replacing each vertex of $G$ by an independent set of vertices, and each edge of $G$ by a complete bipartite graph between sets corresponding to its ends. If all sets replacing vertices are of the same size $p$ the blown-up copy of $G$ is denoted by $G^{p}$. If $G$ is edge-colored then we call a colored graph $G^{p}$ the true colored copy of $G$ if all edges of the bipartite part corresponding to a given edge of $G$ have its color. 
Theorem 2 Builder wins the Ramsey on-line game on the class of $k$-colorable graphs with any $k$-colorable graph as a target.

Proof: Let $p, k>1$ be fixed integers. It suffices to show that Builder can force the complete $k$-partite graph $K_{k}^{p}$. Let $n=n(k, k, k)$ be as defined in the proof of Theorem 1 . Let $m$ be large enough so that Builder can force Painter to create a monochromatic $K_{k}$ while building a $k$ colorable graph on $n$ vertices and $m$ edges. Let $V_{1}, \ldots, V_{n}$ be disjoint sets of vertices of size $B^{(m)}(p)$ and let $V=V_{1} \cup \ldots \cup V_{n}$. Let $U=\left\{v_{1}, \ldots, v_{n}\right\}$ be another set disjoint from $V$. Builder will apply her strategy from the $K_{k}$-game while constructing bipartite graphs between sets $V_{i}$, using $U$ as an auxiliary board in the following way. Let $p_{i}=B^{(m-i)}(p)$ for $i=1, \ldots, m$. At the first stage she presents all edges between $V_{1}$ and $V_{2}$. By the Bipartite Ramsey Theorem a monochromatic copy of $K_{p_{1}, p_{1}}$ appears. This is marked on the auxiliary board $U$ by painting the edge $v_{1} v_{2}$ the same color. Assume by induction that after the $i$-th stage of the game the situation looks as follows: (1) there is a red-blue graph $G_{i}$ on $U$ which is a winning position for Builder in the $K_{k}$-game, and (2) the graph $H_{i}$ constructed on $V$ is a blown-up copy of $G_{i}$ containing the true colored copy $G_{i}^{p_{i}}$ of $G_{i}$. Let $X_{1}, \ldots, X_{n}$, with $\left|X_{i}\right|=p_{i}$ be the independent parts of the graph $G_{i}^{p_{i}}$ corresponding to vertices $v_{1}, \ldots, v_{n}$ of $G_{i}$. Note that $G_{i}$ and $H_{i}$ are $k$-colorable graphs. Now, suppose $e=v_{r} v_{s}$ is the next edge Builder would present if she had played the $K_{k^{-}}$ game on $U$. Then in the actual game on $V$ she presents all edges between $X_{r}$ and $X_{s}$ to get a monochromatic copy of $K_{p_{i+1}, p_{i+1}}$, and next paints $e$ with the same color. Thus $G_{i+1}=G_{i}+e$. To see that this completes the inductive step let $Y_{r} \subset X_{r}$ and $Y_{s} \subset X_{s}$ be the bipartite parts of the just obtained monochromatic $K_{p_{i+1}, p_{i+1}}$ and let $Y_{j} \subset X_{j}$, $j \neq r, s$, be arbitrary sets of size $p_{i+1}$. Then the graph $G_{i+1}^{p_{i+1}}$ induced by $Y_{1} \cup \ldots \cup Y_{n}$ is a true colored copy of $G_{i+1}$. By the choice of $m$ the proof is complete.

\section{Planar graphs}

Another class of graphs with this peculiar self-unavoidability property is the class of forests.

Proposition 1 Builder can force Painter to create any monochromatic forest in the Ramsey on-line game on forests.

Proof: It suffices to prove the assertion for trees. Let $T$ be any tree with $n$ vertices $v_{1}, \ldots, v_{n}$. We apply induction on the number of vertices. Let $u$ be a leaf of $T$ and let $T^{\prime}=T-u$. By induction Builder can force $n$ disjoint copies $T_{1}, \ldots, T_{n}$ of $T^{\prime}$ in the same color, say red. (See Figure 1 where $T$ is a binary tree of height 2.) Let $u_{i}$ be the vertex of $T_{i}$ corresponding to the neighbor of $u$ in $T$. Now, Builder shows exactly those edges $u_{i} u_{j}$ for which the corresponding pair $v_{i} v_{j}$ is an edge of $T$. Since Painter cannot paint it red, a blue copy of $T$ will appear. Clearly, no cycles have emerged on the painted edges. 


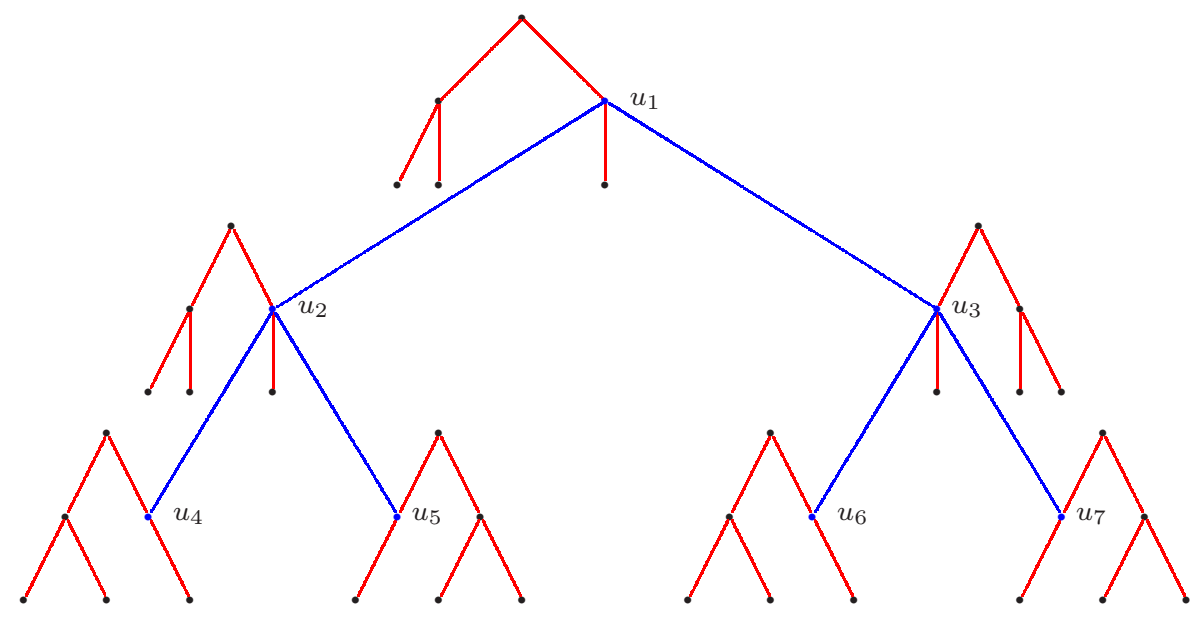

Figure 1: Forcing a tree

A non-trivial example in the opposite direction can be found already in the class of outerplanar graphs (that is, planar graphs having a plane embedding with all vertices lying on the outer face).

Theorem 3 Painter can avoid a monochromatic triangle on outerplanar graphs.

Proof: We will show that Painter can control the number of red and blue edges so that their difference on any bounded face is not divisible by 3 . We argue by induction. Suppose that in the first $n$ moves an outerplanar graph $G$ satisfying the requirement has been constructed, and let $e$ be an edge shown by Builder in the next move. Then either 0,1 or 2 new bounded faces can be created. The first two possibilities are trivial. In the last situation $e$ must cross a bounded face $F$ (by outerplanarity of $G$ ) splitting its boundary cycle into two paths, say $P_{1}$ and $P_{2}$. Let $r_{i}$ and $b_{i}$ denote the number of red and blue edges in $P_{i}$, respectively. Then Painter can color $e$ so that both new faces are colored properly, unless $r_{i}-b_{i} \equiv(-1)^{i}$ or $(-1)^{i-1}(\bmod 3)$. In both cases we get $\left(r_{1}+r_{2}\right)-\left(b_{1}+b_{2}\right) \equiv 0(\bmod 3)$ which contradicts our induction hypothesis.

On the other hand, Builder can force a monochromatic triangle while building the edges of a 2-degenerate planar graph.

Proposition $2 K_{3}$ is unavoidable for Painter on planar 2-degenerate graphs.

Proof: Builder starts with forcing 3 disjoint copies $S_{1}, S_{2}, S_{3}$ of a star $K_{1,2}$ in the same color, say red. Let $c_{i}$ denote the center of $S_{i}, i=1,2,3$. Now Builder joins $c_{i}$ with the leaves of $S_{i+1}$ in the cyclic order mod 3 . Then either a red-blue diamond or a red $C_{6}$ without a chord appears. The first case allow Builder to finish the game in one move while the second in the next three moves, by joining each second vertex of $C_{6}$ (see Figure 2 ). It is not hard to check that the resulting graph is planar and 2-degenerate. 


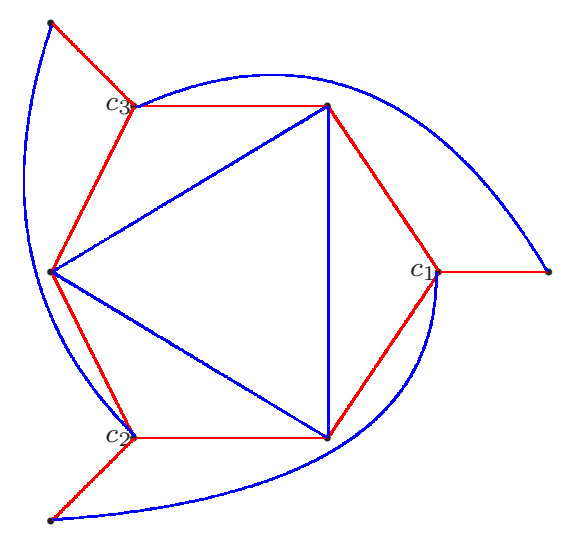

Figure 2: Forcing a triangle

In general, any cycle is unavoidable on planar graphs.

Proposition 3 Cycles are unavoidable for Painter on planar graphs.

Proof: To force $C_{n}$ Builder adds edges incident to a vertex $x$ until she has a large monochromatic (say red) star with center $x$ and leaf set $L$. Now playing on $L$ she builds a forest to force a monochromatic path $P$ with $t=(n-2)(n-1)$ edges. So far the graph can be drawn with $x$ and $P=v_{0} v_{1} \ldots v_{t}$ on the outer face. If the path is red we are done, so assume that it is blue. Now Builder shows $n-2$ edges of the path $v_{0} v_{n-1} v_{2(n-1)} \ldots v_{t}$, and if any of these is painted blue we are done. Otherwise we get a red path on $n-1$ vertices, which together with vertex $x$ form a red $C_{n}$.

We suspect that Painter should be able to avoid $K_{4}$, and maybe even $K_{2,3}$, on planar graphs, but so far we haven't found a proof of either of these. The next Proposition demonstrates that these questions are on the edge of our knowledge.

Proposition 4 The graph $K_{4}-e$ is unavoidable for Painter on planar graphs.

Proof: To force $K_{4}-e$ Builder adds edges incident to a vertex $x$ until she has a monochromatic (say red) star with center $x$ and 9 leaves $v_{0}, \ldots, v_{8}$. Next she adds edges $v_{0} v_{i}$ for $i \in[8]$. To avoid a red $K_{4}-e$ Painter must color at least 7 (say $v_{0} v_{i}$ for $i \in[7]$ ) of them blue. Now Builder adds the edges $v_{2 i-1} v_{2 i}$ for $i \in[3]$ and Painter must color two of them (say $v_{2 i-1} v_{2 i}$ for $i \in[2]$ ) with the same color (say blue), as in Figure 3. Finally, Builder adds the edges $v_{7} v_{2}$ and $v_{7} v_{3}$. If Painter colors them both red then $\left\{x, v_{0}, v_{2}, v_{3}, v_{7}\right\}$ induces a red $K_{4}-e$; otherwise there is a blue $K_{4}-e$ induced by a set of the form $\left\{x, v_{0}, v_{2 i-1}, v_{2 i}, v_{7}\right\}$. 


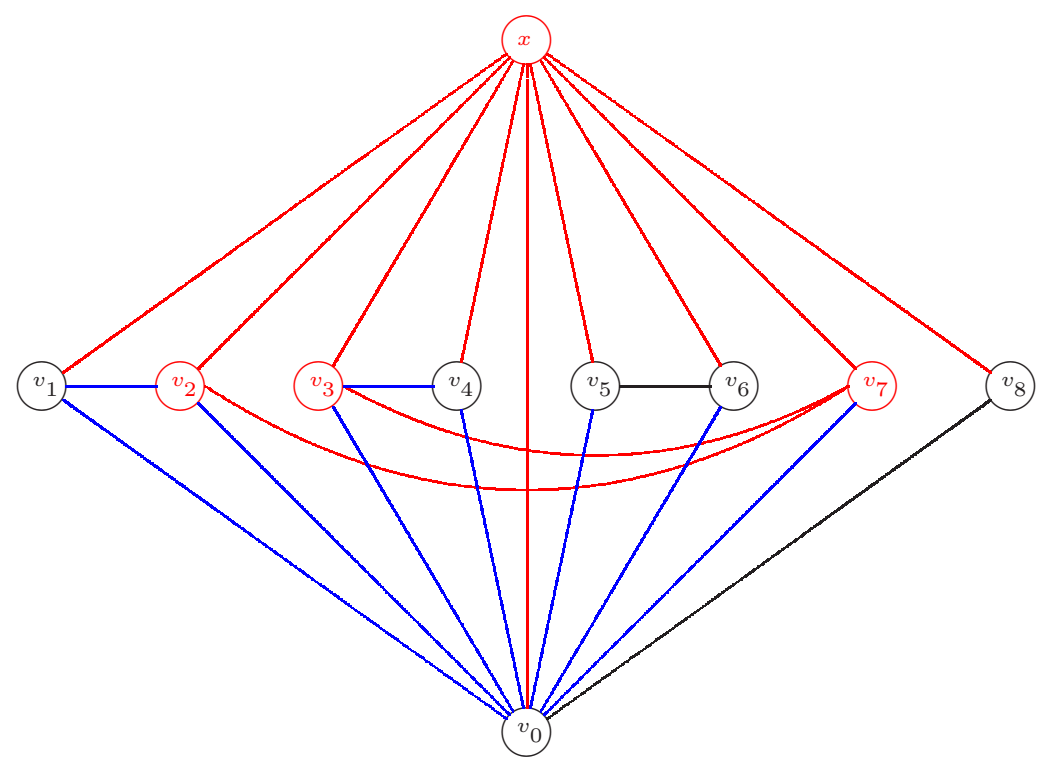

Figure 3: Forcing $K_{4}-e$

\section{More colors}

It is natural to expect that the results of previous sections are valid if Painter has any fixed number of colors at his disposal. However, with the exception of Proposition 1, their proofs do not seem to generalize in the obvious way. Below we prove a multicolored version of Theorem 1 for $k=3$ using the following auxiliary game.

Let $r, s \geq 2$ be fixed integers. The $(r, s)$-survival game is defined as follows. Alice plays by presenting $r$ vertices to Bob. Bob plays by putting an edge between two of them and discarding the rest. These discarded vertices just disappear together with all edges incident with at least one of them. Alice can never again present a vertex that Bob has discarded. Alice's goal is to create a clique of size $s$ while Bob will try to prevent her from doing so.

Lemma 1 For any $r, s \geq 2$ there exists $t=t(r, s)$ such that Alice can create a clique of size $s$ in a graph with at most $t$ vertices, while playing the $(r, s)$-survival game with Bob.

Proof: We apply induction on $s$ with $r$ being fixed. For $r=2$ the assertion is trivial with $t(2, s)=s$. Assume the lemma holds for $s-1$ and let $d=t(r, s-1)$. It suffices to show that Alice can create an induced star $S$ with center of degree $d$, since then she can continue on the leaves of $S$ to get $K_{s-1}$ there. Assume by induction that Alice can create an induced star with $d-1$ leaves. Repeating this construction $r$ times on independent sets of vertices she gets $r$ disjoint stars with centers $v_{1}, \ldots, v_{r}$ of degree $d-1$. Then in her last move she presents this set of centers and obtains a desired star $S$.

Let $R_{c}(n)$ be the multicolored Ramsey number, that is, the minimum $R$ such that in any $c$-coloring of the edges of $K_{R}$ there is a monochromatic copy of $K_{n}$. 
Theorem 4 Builder can force Painter to create a monochromatic triangle in the multicolor Ramsey on-line game while playing on a 3-colorable graph.

Proof: We apply induction on the number of colors $c$. The assertion is trivial for $c=1$, so let $c \geq 2$, and assume it holds for $c-1$ colors. Let $n$ be the number of vertices of a 3-colorable graph $F$ on which Builder wins against Painter armed in $c-1$ colors. Let $r=c+1, s=R_{c}(n)$ and let $m$ be large enough so that Alice can creates $K_{s}$ in $m$ moves of the $(r, s)$-survival game. (For the rest of the proof assume that Builder's name is Alice and Painter's name is Bob.) Let $X, Y$ and $U$ be disjoint sets of vertices of size at least $\mathrm{rm}$. As in the proof of Lemma 1, $U$ will serve as an auxiliary board on which Builder will mark Painter's moves translated into Bob's moves in the game of survival. For this purpose let $f: Y \rightarrow U$ be a bijection. In the first part of the game Builder constructs a bipartite graph between $X$ and $Y$ as follows. In the first move she picks a set $A_{1} \subset Y$ of $r$ vertices and joins them to a vertex $a_{1}$ in $X$. Since $r>c$ at least two edges $a_{1} u_{1}$ and $a_{1} v_{1}$ will get the same color, say red. Then she joins their images $f\left(u_{1}\right)$ and $f\left(v_{1}\right)$ by a red edge and discards the rest of $f\left(A_{1}\right)$. In the next move she picks a set $A_{2} \subset Y$ of size $r$ such that $f\left(A_{2}\right)$ is the next move in her strategy for the $(r, s)$-survival game on $U$ and joins its vertices to a new vertex $a_{2} \in X$. After Painter's move there will be two edges $a_{2} u_{2}$ and $a_{2} v_{2}$ of the same color which gives Bob's next move in the $(r, s)$-survival game. And so on. By Lemma 1, after at most $m$ moves of this type there will be $c$-colored $K_{s}$ on $U$ containing a monochromatic clique $K_{n}$. Assume the clique is red and $V$ is the set of its vertices. The game will now be continued on the set $f^{-1}(V) \subset Y$. Since no edge of $f^{-1}(V)$ can be painted red, Builder can apply her strategy for $c-1$ colors to get a monochromatic triangle in a 3-colorable graph $F$.

We are left to show that the whole resulting graph is 3-colorable. Let $G$ be the bipartite graph between $X$ and $Y$ constructed in the first part of the game. We may assume that there are no isolated vertices in $G$. Let $H$ be the corresponding auxiliary graph on the set of vertices $S \subset U$ that survived till the end of this part. We claim that if $F$ is any 3-colorable graph with vertex set $V(F)=f^{-1}(S)$ then any proper 3-coloring of $V(F)$ can be extended to a proper 3-coloring of the whole graph $G \cup F$. Let $a_{i} \in X$ and $A_{i} \subset Y$ be the vertices picked in the $i$-th step. Consider the following relation $\rho$ on the set $Y$ : $(a, b) \in \rho$ iff there exists $j$ such that $a, b \in A_{j}, f(a)$ is discarded in step $j$ and $b$ is the largest element (under some total ordering of $Y$ ) of $A_{j}$ such that $f(b)$ survives step $j$. Let $\preceq$ denote the transitive and reflexive closure of $\rho$. Clearly $\preceq$ is a partial ordering. Moreover, for each vertex $y \in Y$ there exists a unique maximal element $z_{y}$ such that $y \preceq z_{y}$. Note that $f\left(z_{y}\right)$ is a surviving element, and so $z_{y} \in V(F)$. Color $y$ with the same color as $z_{y}$. So far this is safe since the discarded vertices are isolated in $F$. Now consider a vertex $a_{i} \in X$ with neighborhood $A_{j}$. Suppose that $f\left(b^{\prime}\right)$ and $f(b)$ are the surviving elements of the $i$-th step with $b^{\prime}$ smaller than $b$. Then all the vertices of $A_{i}$ have the same color as $b$, except possibly $b^{\prime}$. Thus there is a third color available with which to color $a_{i}$. This enables us to extend the original 3-coloring of $F$ to a proper 3-coloring of the whole graph. The proof is now complete. 
The following corollary can be deduced from the Bipartite Ramsey Theorem, similarly as Theorem 2.

Corollary 1 Builder wins the multicolor Ramsey on-line game on the class of 3-colorable graphs with any 3-colorable graph as a target.

The above method does not seem to be easily extendable to the general case of $k$ colorable graphs. One natural attempt leads to the more general version of the $(r, s)$ survival game (see 5.3).

\section{Problems}

\subsection{Monotone classes of graphs}

The most ambitious problem for further investigation would be to characterize selfunavoidable classes among monotone decreasing classes of graphs. In particular, it would be interesting to know the truth in case of the most popular families of graphs. For instance, we expect a negative answer for planar graphs, but curiously, it is not so easy to construct an example of Painter's win in this class. It is also tempting to conjecture that perhaps outerplanar graphs are unavoidable while other planar graphs are not.

Conjecture 1 The class of graphs unavoidable on planar graphs is exactly the class of outerplanar graphs.

Let $\mathcal{G}_{k}$ be a family of graph classes indexed by some graph parameter $k$ (thickness, genus, treewidth, degeneracy, colorability, etc.). Let $f(k)$ be the minimum number such that any graph $H \in \mathcal{G}_{k}$ is unavoidable in the Ramsey on-line game on $\mathcal{G}_{f(k)}$. It would be interesting to study the speed of $f(k)$ for the most important graph parameters. For many of them it is not even clear whether $f(k)$ is finite for all $k$.

\subsection{Ramsey on-line numbers}

Let $\mathcal{G}_{m}$ be the class of graphs with at most $m$ edges. The on-line Ramsey number $\bar{R}(k)$ is the minimum $m$ such that $K_{k}$ is unavoidable on $\mathcal{G}_{m}$. The problem of estimating $\bar{R}(k)$ was posed in [1] and [2]. It is obvious that $\bar{R}(k)$ is at most $\left(\begin{array}{c}R(k) \\ 2\end{array}\right)$, where $R(k)$ is the usual Ramsey number. Also it is not hard to prove that $\bar{R}(k)$ is at least $R(k) / 2$ (see [1). Improvement of these bounds may appear hard, as it is not even clear if $\bar{R}(k)=o\left(R(k)^{2}\right)$ (see [2, 4]).

\subsection{Ramsey game of survival}

Consider the following generalization of the $(r, s)$-survival game presented in section 4 . Let $r, s, k$ be fixed integers with $k \geq 2$ and $r, s \geq k$. In one round of the $(r, s, k)$-survival game Alice presents $r$ vertices and Bob discards $r-k$ of them. Those $k$ vertices that survive 
(at least to the next round) form a hyperedge. Surviving vertices can be presented in later rounds but a hyperedge with at least one deleted vertex is irretrievably lost. Alice's goal is to create a $k$-uniform complete hypergraph on $s$ vertices. By Lemma 1 she has a winning strategy in case $k=2$ for arbitrary $s$, but can she win this game for any $k>2$ ? A positive answer would yield a strategy for Builder in the multicolored Ramsey on-line game on $k$-colorable graphs.

\section{References}

[1] J. Beck, Achievement games and the probabilistic method, in: Combinatorics, Paul Erdős is Eighty, Bolyai Soc. Math. Stud., vol. 1, (1993), 51-78.

[2] E. Friedgut, Y. Kohayakawa, V. Rödl, A. Ruciński, P. Tetali, Ramsey games against one-armed bandit, Combin. Probab. Comput., 12 (2003), 515-545.

[3] R.L. Graham, B.L. Rotschild, J.H. Spencer, Ramsey Theory, 2nd edition, Wiley, New York, 1990.

[4] A. Kurek, A. Ruciński, Two variants of the size Ramsey number, manuscript. 